\title{
Qué se entiende por Irradiación Fetal
}

\author{
Dres.: Germán Ramírez C. MSc.*, Rodrigo Cifuentes B. MD, PhD**
}

\section{RESUMEN}

Durante muchos años y en diferentes publicaciones científicas se presentan tablas y estudios que analizan los daños al embrión o feto, cuando la madre es irradiada en las primeras semanas de embarazo y aun durante toda la gestación, como es el caso de las madres ocupacionalmente expuestas a las radiaciones. Pero estas publicaciones no le dan al ginecólogo una idea clara de cómo la radiación daña las células del feto y qué determina el alto riesgo a la irradiación. Debido a la alta concentración de energía que transporta la radiación ionizante, de cualquier tipo, en especial la radiación gamma y los rayos $\mathrm{X}$, de amplio uso en medicina, los centros vitales de las células, como cromosomas y el ADN sufren daños en muchos casos irreversibles cuando las células están en proceso de mitosis, que es el caso del feto en las primeras semanas del embarazo. Se presenta en este trabajo una descripción completa del agente dañino, "LA RADIACION IONIZANTE", la manera en que ella interactúa con las células y los factores que determinan la alta radiosensibilidad del feto.

\section{INTRODUCCION}

A partir del año 1889 en que William Roentgen descubrió los rayos X y comenzó su uso médico, muchas poblaciones se han visto expuestas a las radiaciones ionizantes, convirtiéndose el uso médico y odontológico en el mayor contaminante de radiación a la humanidad. Este alto uso y en ocasiones indiscriminado de las radiaciones ionizantes, deter-

* Física - Médica, Radiofísica Hospital Universitario del Valle;

** Jefe Sección de Obstetricia, Facultad de Salud Universidad del Valle. mina en algunos casos que se altere el funcionamiento de los organismos vivos debido a daños en las estructuras moleculares inducidas por la interacción de la radiación con la materia. Una de las poblaciones más críticas en cuanto a exposición a las radiaciones es la de los embriones y fetos debido al alto grado de mitosis que está ocurriendo en estos organismos. Desafortunadamente muchas madres en las primeras semanas de embarazo se ven sometidas a estudios radiográficos en los cuales queda incluido el abdomen determinando esto algunas veces altas dosis al feto que a la luz de las múltiples investigaciones realizadas en este campo determinan un mayor riesgo de anormalidades en el feto o inducción de neoplasias en el niño.

El agente responsable de este tipo de alteraciones celulares o moleculares es la radiación ionizante, la cual es un medio físico que transporta energía, en unos casos en forma de partículas y en otros en forma de energía pura, como es el caso de la radiación Gamma y los Rayos X. La energía que transporta la radiación es generalmente depositada en los organismos por medio de interacciones de ésta con las moléculas presentes en nuestro organismo y en especial, en las moléculas más sofisticadas, donde existe una mayor probabilidad de que se rompan los enlaces entre los diferentes elementos que forman estas moléculas.

\section{Teorías:}

La radiología considera que en la célula existen centros vitales que se convierten en blancos de la radiación y los cuales al ser atacados por ésta, determinan la alteración de las funciones celulares o la muerte de la célula. El centro vital y de alta radiosensibilidad que se ha determinado en las modernas teorías de radiobiología, es el núcleo de la célula. 
Lo que determina la alta radiosensibilidad del núcleo celular son las moléculas tan especializadas presentes y las funciones tan específicas y vitales para la normal subdivisión celular, proceso esencial para la supervivencia celular. Las moléculas de ácidos desoxirribonucleico, DNA y ribonucleico ARN, constituyen moléculas altamente sofisticadas en cuanto a su composición y formas heliocoidales (Figura 1). Los múltiples enlaces entre los azúcares, azufres y bases, determinan códigos específicos y pregrabados en la molécula, de manera que cada célula, reconocerá sus códigos de subdivisión celular y genéticos a través de la interpretación de dichos códigos. Por lo anterior se define a la molécula de ADN como el director de orquesta en la célula.

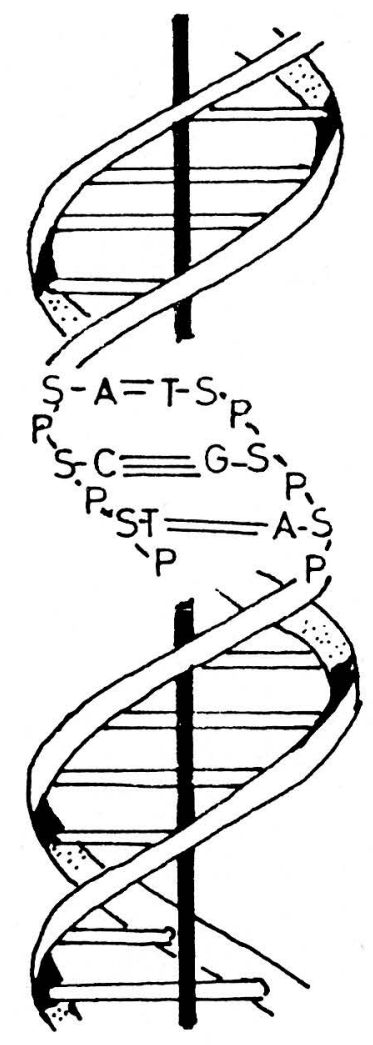

Fig. 1. Estructura tridimensional del ADN, S, P, A, T, G, C, representa azúcar, fósforos, adenina, timina, guanina y citonina respectivamente.

La radiación que es el agente dañino o destructor de la célula o moléculas, es un fenómeno físico presente en el universo desde hace miles de años. Una de las fuentes más conocida y espectacular de radiación ionizante es el sol que nos alumbra. En esta estrella existe una permanente emisión de partículas radioactivas debido a las explosiones termonucleares que continuamente se suceden en su superficie y que diariamente llegan a la superficie de la tierra, sumándose a la radiación existente en los diferentes elementos radioactivos presentes en ésta. También contribuyen a la radiación ambiental a la cual estamos sometidos diariamente, las pantallas de televisión, las diferentes emanaciones de las paredes de nuestras viviendas o edificaciones, objetos construidos con material terrestre como vasos y muy especialmente en los últimos años el cigarrillo debido a la avidez con que las hojas de tabaco toman el Polonio-210 elemento radioactivo que el hombre ha liberado a la atmósfera en gran cantidad.

La radiación ionizante se puede describir como la generada en el núcleo de átomo o atómica y la generada a nivel orbital o por interacción de partículas con las diferentes órbitas del átomo o acción externa del núcleo. La manera como cualquiera de estos tipos de radiacción interactúa o deposita su energía en las moléculas está descrito, físicamente por los efectos fotoeléctrico, compton y formación

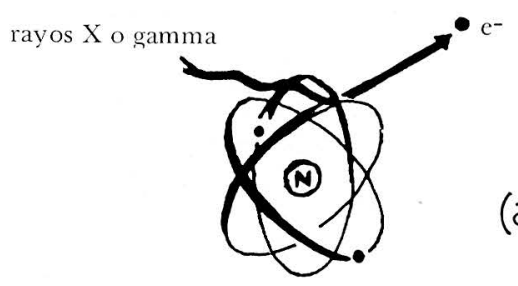

(a)
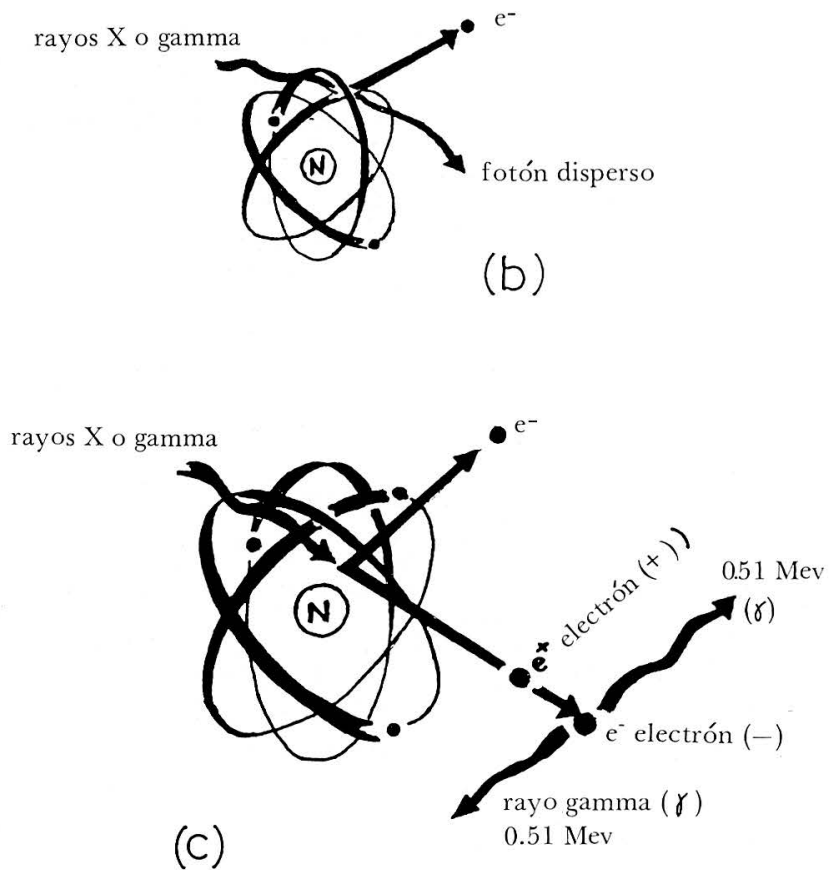

Fig. 2. Interacción de los rayos X y gamma con la materia. (a) Efecto Fotoeléctrico. (b) Efecto compton. (c) Formación de pares. 
de pares, efectos que son claramente dependientes de la energía que transporta la radiación (Figura 2).

Finalmente se han formulado dos teorías de la manera como la radiación interactúa con la célula, fenómeno que por suceder en un período de tiempo menor a $10^{-16} \mathrm{seg}$. ha hecho imposible determinar exactamente lo que sucede en esta interacción. Estas dos teorías son las de acción directa y la de acción indirecta. La acción directa considera que existe un centro vital en la célula, donde la radiación incide como un proyectil destruyendo los enlaces de las moléculas vitales como la de ADN (Figura 3). La acción indirecta considera que los centros vitales de la célula son atacados por partículas formadas en los medios celulares que rodean las moléculas vitales. Generalmente se considera que estas partículas son iones formados en los medios acuosos grandes en la célula, (Figura 4).

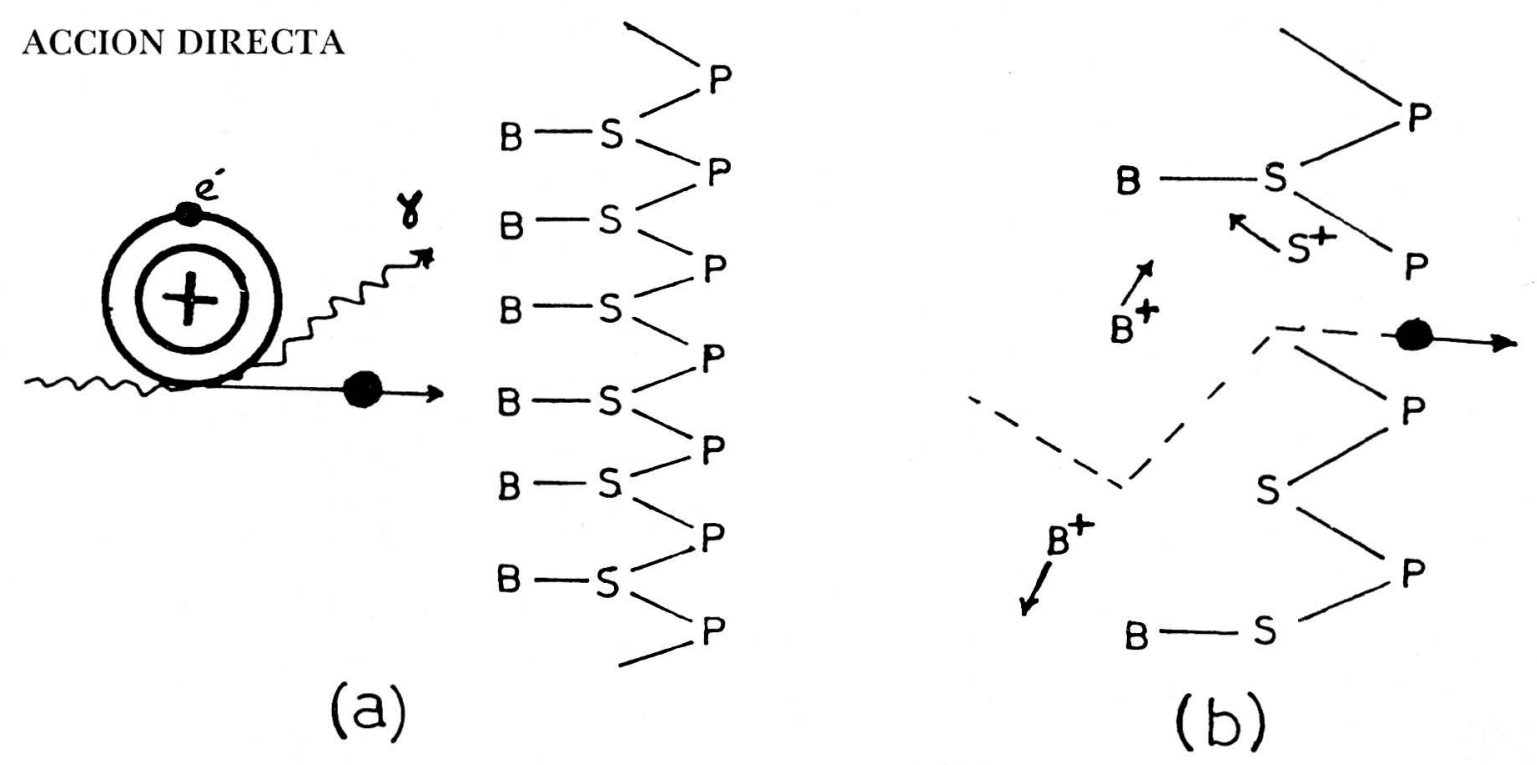

Fig. 3. a) La radiación incidente extrae un electrón de los átomos vecinos a la molécula de ADN. b) El electrón actúa como proyectil que rompe uno o varios enlaces a su paso por la molécula de ADN. $\mathrm{B}=$ Base, $\mathrm{S}=$ Azúcares y $\mathrm{P}=$ Fosfatos.

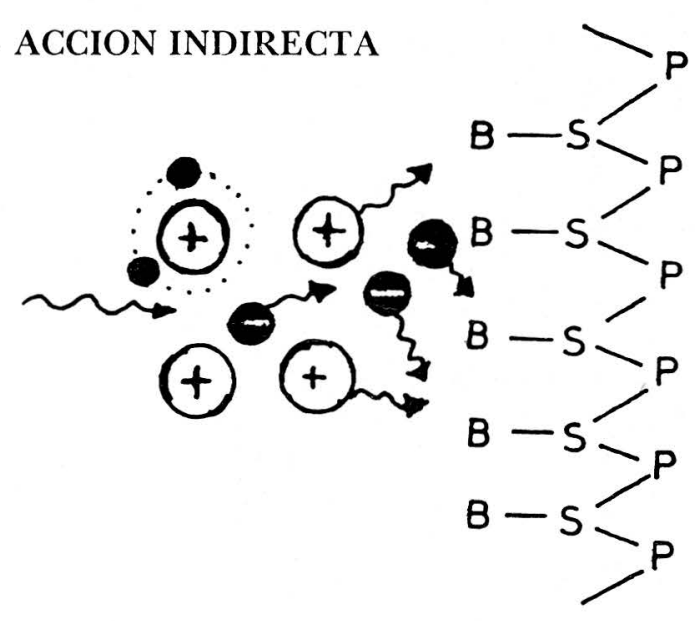

(a)

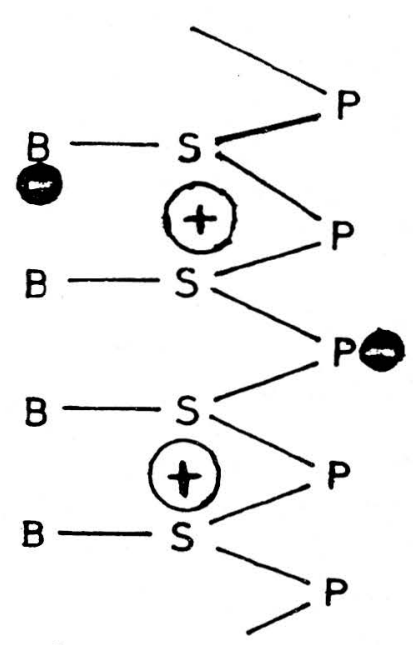

(b)

Fig. 4. a) La radiación incidente rompe enlaces moleculares en la vecindad de la molécula de ADN creándose iones altamente reactivos. b) Los iones reaccionan con la molécula de ADN, transformándola químicamente. 


\section{Investigación}

Muchos autores $(1,2,3,4,5)$ han realizado múltiples investigaciones, para obtener una respuesta de los daños biológicos y genéticos que se pueden inducir al irradiar tejidos biológicos. Desafortunadamente es imposible hacer experimentos en seres humanos vivos, debido a los daños irreversibles que se pueden inducir en estos. La información que han podido obtener los investigadores, es analizando poblaciones que accidentalmente o en desafortunados eventos de la humanidad han sido expuestos a irradiación. Las poblaciones más clásicas son:

Los sobrevivientes de Hiroshima y Nagasaki, niños tratados por hipertrofia del timus con radiación, adultos que fueron tratados con rayos $\mathrm{X}$ de espondilitis anquilosante a la columna, adultos tratados con iodo radioactivo para problemas benignos de la glándula tiroides, mujeres que ingirieron Radium-224 cuando pintaban los números de tableros de relojes, trabajadores en minas de material radioactivo y niños expuestos a rayos $\mathrm{X} u$ otras fuentes de radiación en útero.

Otros estudios se han conducido en animales y su información se ha extrapolado a los humanos, sin ser muy clara su relación de dosis-peso y otras variables fisiológicas, que difieren de una especie a otra.

Las investigaciones de los físicos han demostrado claramente que la radiación se clasifica en radiación alfa (Figura 5-a) la cual está constituida por dos protones y dos neutrones que son expulsados de los núcleos pesados de los átomos; radiación beta (Figura 5-b) la cual está formada por un electrón positivo o negativo que se formó cuando un neutrón se convirtió en un protón o un protón en un neutrón, procesos que ocurren dentro del núcleo, lo cual determina que el electrón también sea expulsado de dentro del núcleo. Debido a las dos emisiones antes mencionadas, se genera una energía dentro del núcleo la cual también es expulsada en forma de cuantos, paquetes o fotones (Figura 5-c), de energía y se denomina radiación gamma de gran uso en medicina. Los rayos $\mathrm{X}$ utilizados en diagnóstico, se forman a partir de un electrón que atravieza el campo eléctrico formado por los electrones en las capas del átomo e interactúa con el núcleo pasando muy cerca de él, cambiando su trayectoria y perdiendo parte de su energía, la cual se constituye en los rayos $\mathrm{X}$ duros o de frenado (Figura 5-d).

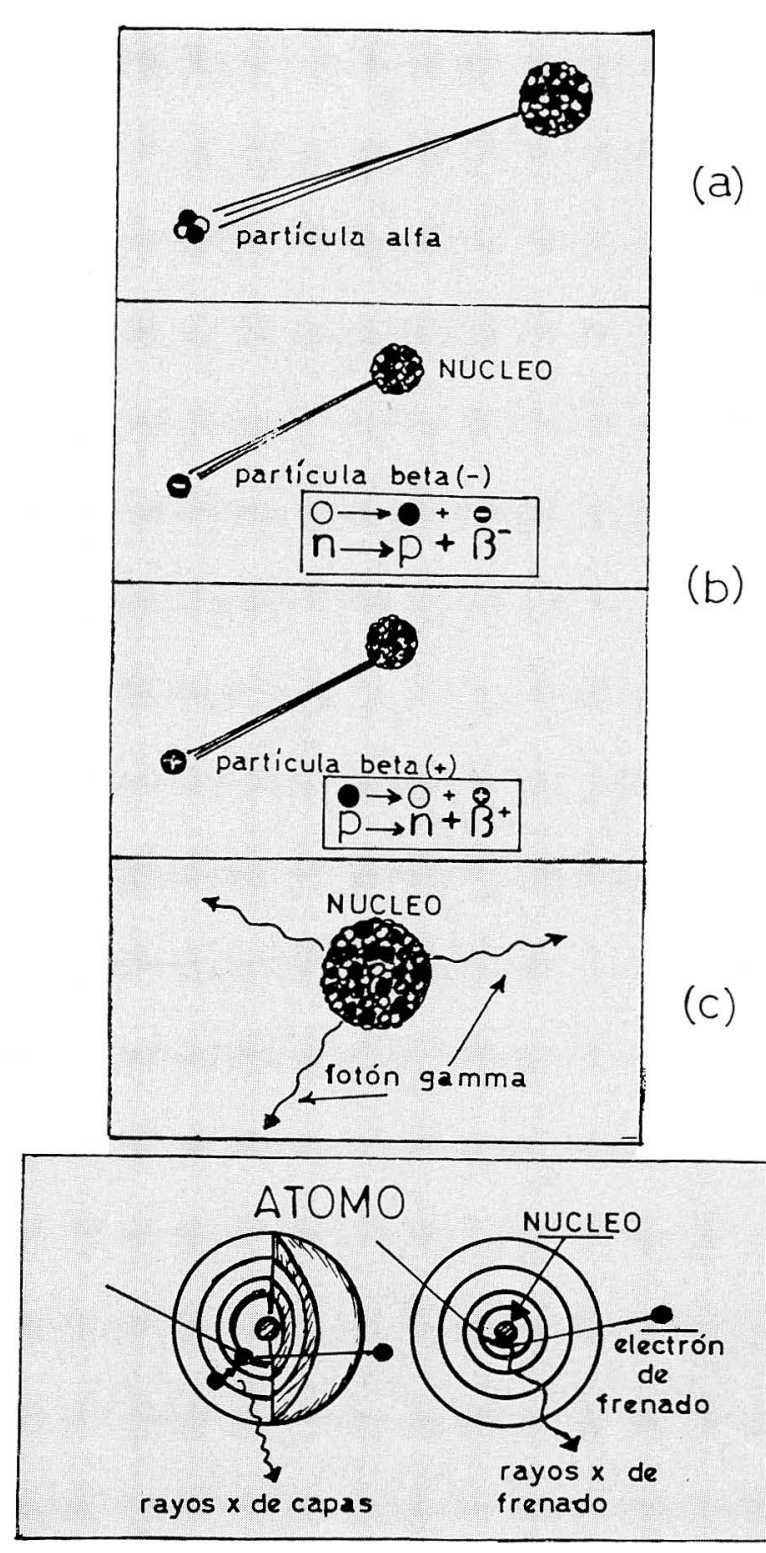

(d)

Fig. 5. Tipos de radiación ionizante. a) Radiación alfa. b) Radiación beta negativa y positiva. c) Radiación gamma. d) Rayos X duras y de capas.

La biología molecular ha determinado con exactitud los componentes, forma y funciones de las moléculas de ADN y ARN. Experimentos en los cromosomas, determinaron que si se irradiaban células con cromosomas normales, se inducía en ellas una desordenada multiplicación de éstos, hasta llegar a convertirse en células cancerosas. También se demostró que al irradiar células normales y revisar mediante un extendido los pares cromosómicos se inducía alteraciones cromosómicas en estas células. Estos dos experimentos determinaron que existe una correlación entre inducción del cáncer, aberra- 
ciones cromosómicas y radiación ionizante. La pregunta que se hacía la biología celular es si todas las células tienen la misma respuesta de daño o deterioro ante la radiación. La respuesta que ellos han encontrado es que existe una relación entre el proceso de subdivisión celular y la radiosensitividad de la célula. Está plenamente demostrado que las células en mitosis son más sensibles a la radiación y células en profase y metafase son también más sensibles que en anafase y telofase.

En la tabla 1 se da la clasificación en orden descendente de radiosensitividad celular.

TABLA 1

RADIOSENSITIVIDAD CELULAR

Tabla 1. Podemos notar que células con procesos de mitosis cortos, presentan una mayor radiosensitividad, que aquellas con mitosis largas o únicas como son las células del SNC.

En la tabla 2 se presenta la radiosensitividad de los órganos a la radiación.

Los procesos físico-químicos y bioquímicos que ocurren cuando los seres vivos se ven expuestos a radiaciones ionizantes se presenta en la Figura 6.

\section{DISCUSION}

Los episodios embriogenéticos son continuos y se superponen en el tiempo. Sin embargo, para facilitar su descripción, se han agrupado de tal manera que el desarrollo embriofetal resulta dividido en las siguientes etapas:

1. Período pre-embrionario desde la fecundación hasta el día 20 en que termina la implantación.

2. Período embrionario desde el día 21 al 56 (4a. a 8 a. semana), comprende los organogénesis y morfogénesis.

3. Período fetal, desde el día 57 al 266, (9a. a 38a. semana). Caracterizado por su histogénesis y crecimiento corporal.

A la luz de los expcrimentos y teorías existentes en biología celular, se determina que el período de mayor riesgo para el feto está dentro del primer trimestre y por extrapolación de experimentos en animales alrededor del día 35 post concepción, riesgo debido al alto nivel de organogénesis o subdivisión celular. La clasificación de riesgos presentados en la Figura 7 da una clara idea de los riesgos por irradiación fetal y período de concepción.

TABLA 2

RADIOSENSITIVIDAD RELATIVA DE LOS TEJIDOS

\begin{tabular}{|c|c|}
\hline Radiosensitividad Relativa & Tejido \\
\hline Alta & $\begin{array}{l}\text { Organos linfáticos } \\
\text { Testículos } \\
\text { Ovarios } \\
\text { Intestino }\end{array}$ \\
\hline Relativamente Alta & $\begin{array}{l}\text { Cristalino } \\
\text { Estómago }\end{array}$ \\
\hline Media & $\begin{array}{l}\text { Capilares } \\
\text { Hueso inmaduro } \\
\text { Cartílago inmaduro }\end{array}$ \\
\hline Relativamente Baja & $\begin{array}{l}\text { Hueso maduro } \\
\text { Cartílago maduro } \\
\text { Glándula salival } \\
\text { Organos respiratorios } \\
\text { Riñones } \\
\text { Hígado } \\
\text { Páncreas } \\
\text { Tiroides } \\
\text { Adrenales } \\
\text { Pituitaria }\end{array}$ \\
\hline Baja & $\begin{array}{l}\text { Músculos } \\
\text { SNC }\end{array}$ \\
\hline
\end{tabular}

Tabla 2: Se nota que órganos con células de proceso de mitosis cortos como los Hematopoyéticos y Gónadas, constituyen los organismos más radiosensibles, mientras que las células de músculos, cerebro y $\mathrm{SNC}$, constituyen el grupo más radiorresistente. 
DAÑOS BIOLOGICOS POR RADIACION

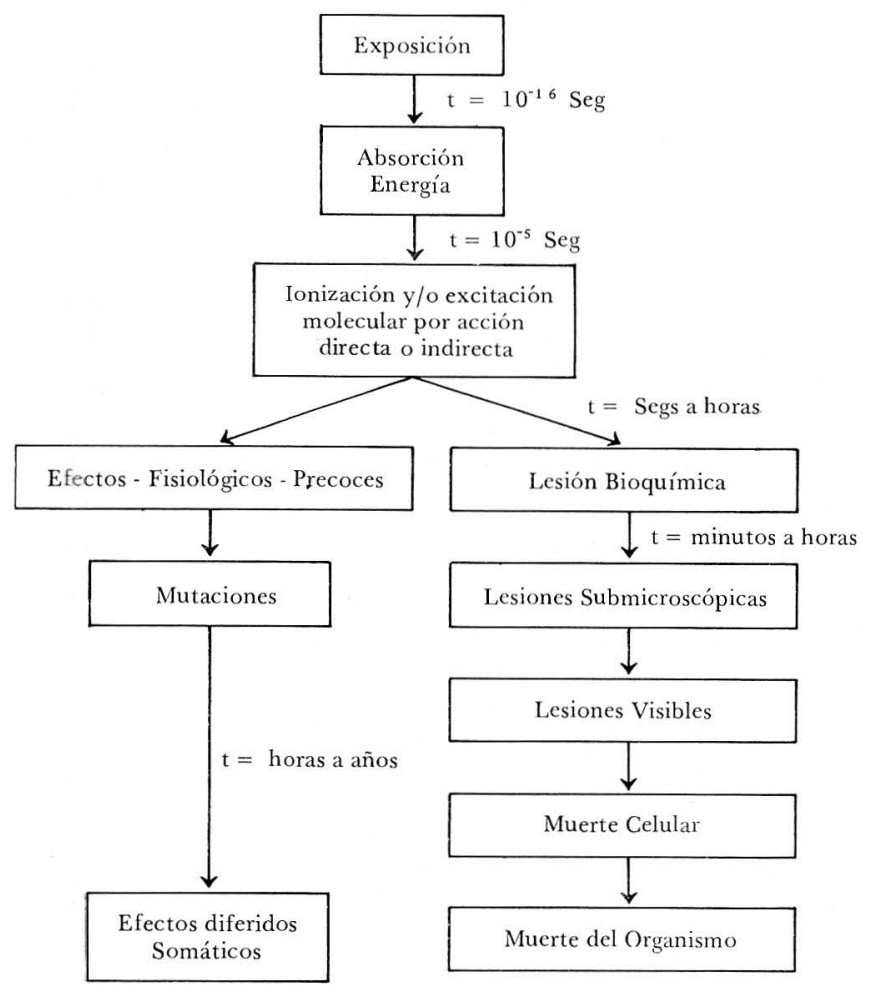

FIGURA 6

\section{CONCLUSIONES}

La radiación como agente externo al feto y la madre, constituye un medio de alteración y deterioro celular de cuidado, por los daños irreversibles e irreparables en el embrión o en el feto, bien sea por alteración en los cromosomas o en el desarrollo normal y diferenciación de las células o por su interferencia con el desarrollo normal de los diferentes órganos que se están formando o desarrollando durante todo el período de la gestación.

La humanidad necesita seguir investigando acerca del comportamiento cromosómico y de sus constituyentes, así como la manera en que la radiación interactúa con las células, cromosomas, moléculas y constituyentes de éstas. Tal vez en un futuro se logre entender más claramente en detalle cómo se deposita la energía en las moléculas y de esta manera se logre crear mecanismos de reparación de las moléculas alteradas por radiación.

Debe recordarse que se tiene una responsabilidad al hacer uso de las radiaciones en seres humanos, por los muchos riesgos a que ellos se ven enfrentados ante este agente físico, ya que no se concibe una medicina u odontología modernas sin el uso de las radiaciones ionizantes, sin perder la meta de que siempre el beneficio debe sobrepasar al riesgo.

INCIDENCIA RELATIVA DE ANORMALIDADES

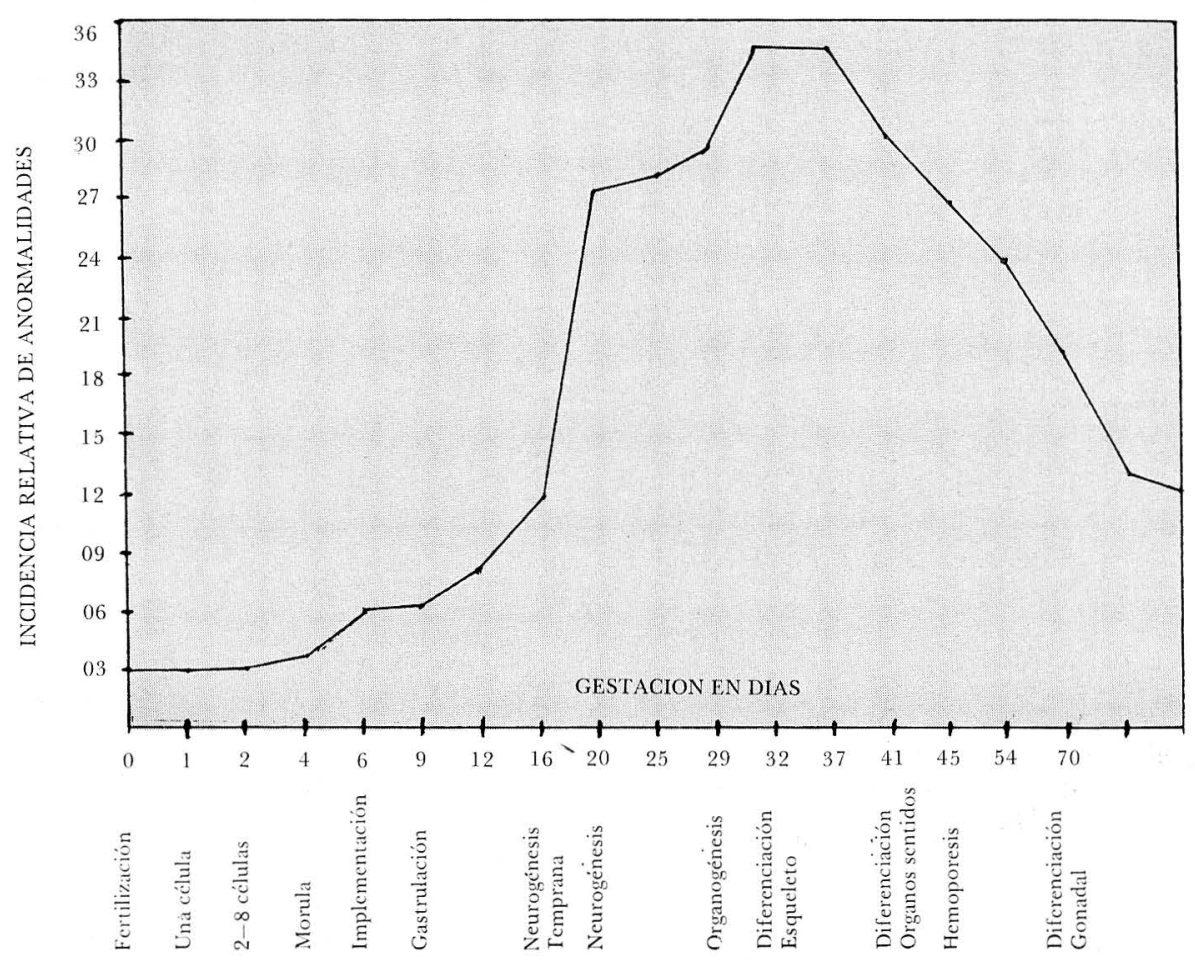

Fig. 7. Teratogénesis en el hombre debido a irradiación con Rayos X. Si las anomalías congénitas se grafican de acuerdo al día de gestación, se ve que en los humanos los días más críticos son del 32 al 37. 


\section{BIBLIOGRAFIA}

1. - REEKIE, D., DAVISON, M., DAVIDSON, J.K.: The radiation hazard in radiography of the female abdomen and pelvis.Brit. J. Radiol. 40, 849, 1967.

2. - MEYER, M.B., TONASCIA, J.A.: Possible effects of $\mathrm{X}$-ray exposure during fetal life on the subsequent reproductive performance of human femalcs. Amer. J. Epidem, 98, 151. 1973.

3. - BLOT, WJ., MILLER, W.: Metal retardation following in utero exposure to the atomic bombs of Hiroshima and Nagasaki. Radiology 106, 617, 1973.

4. - OPPENHEIM, B.E., GRIEM, M.I., MEIER, P.: The effects of diagnostic X-ray exposure on the human fetus: An examination of the evidence. Radiology $114,529,1975$.

5. - RUGH, R.: X-Ray induced teratogenesis in the mouse and its posible significance to man. Radiology 99, 433, 1971.

6. - MOSSMAN, K.L., HILL, L.T.: Radiation risks in pregnancy: The Ameri. College of Obstetricians and Ginecologists 60, No. 2, 237, 1982.

7. - FRIGERIO, N.A.: La radiación y el organismo humano: Comisión de Energía Atómica de USA. Oak Ridge, Tennessee, 1970. 